\title{
Alfven wave modulation of the auroral acceleration region
}

\author{
E. N. Fedorov ${ }^{1}$, V. A. Pilipenko ${ }^{2}$, M. J. Engebretson ${ }^{3}$, and T. J. Rosenberg ${ }^{4}$ \\ ${ }^{1}$ Institute of the Physics of the Earth, B. Gruzinskaya, 10, Moscow 123995, Russia \\ ${ }^{2}$ Space Research Institute, Profsojuznaya 84/32, Moscow 117997, Russia \\ ${ }^{3}$ Augsburg College, 2211 Riverside Ave., Minneapolis, MN 55454, USA \\ ${ }^{4}$ Institute for Physical Science and Technology, University of Maryland, College Park, MD 20742, USA
}

(Received December 1, 2003; Revised July 2, 2004; Accepted July 2, 2004)

\begin{abstract}
We consider the interaction of Alfven waves with the auroral acceleration region (AAR). The AAR is characterized by an electric potential drop that supports a field-aligned upward current and the acceleration of precipitating electrons. An Alfven wave incident on the AAR from the magnetosphere partially reflects back and partially penetrates into the AAR. The rate of wave reflection/transmission is estimated to be critically dependent on the wave transverse scale. Magnetospheric Alfven waves penetrating into the AAR can produce oscillatory variations of the field-aligned potential drop, thus constituting a new mechanism of ULF modulation of electron acceleration. Estimates of the potential drop modulation by Alfven waves are provided within the "thin" AAR approximation, which is valid for a wide range of wave and plasma parameters. The proposed mechanism will produce nearly simultaneous ULF magnetic and riometric variations at auroral latitudes. Occurrence of the AAR-associated resonator in the auroral topside ionosphere between the bottom boundary of the AAR and the E-layer may cause oscillatory frequency dependence of electron acceleration modulations in the range around fractions of a Hz. Another feature of the mechanism considered is the critical dependence of the ratio between the magnetic and riometric signals on the transverse scale of the disturbance. The predicted effects are to be searched for in the simultaneous data of IRIS multi-beam riometers and magnetometers.
\end{abstract}

Key words: Pulsating aurora, ULF waves, riometers, electron acceleration.

\section{Introduction}

The dynamics of ULF waves in Earth's magnetosphere are closely related to that of particles, and various kinds of interrelationships can take place. Magnetospheric MHD waves can modulate effectively the particle distribution function near the magnetospheric equator (Southwood and Kivelson, 1981). Moreover, the interaction of ULF waves with trapped particles can lead to modulated precipitation into the ionosphere. In ground-based studies modulated electron precipitation is commonly revealed with ionospheric riometer observations or pulsating aurora observations. Most current notions about modulation of electron precipitation by ULF waves are based on the mechanism suggested by Coroniti and Kennel (1970), in which the compressional component $b_{\|}$of the wave magnetic field modulates the growth rate of the electron-cyclotron instability, which causes pitch-angle diffusion of electrons into the loss-cone. The idea of quasiperiodic precipitation owing to the interactions of electrons with VLF turbulence was further developed by Davidson (1990) and Demekhov and Trakhtengerts (1994). However, quite often investigation of electron precipitation associated with ULF pulsations did not reveal either compressional pulsations (Nose et al., 1998) or background VLF turbulence (Paquette et al., 1994). Moreover, taking into consideration the ULF wave and electron transit times from an interaction

Copy right(c) The Society of Geomagnetism and Earth, Planetary and Space Science (SGEPSS); The Seismological Society of Japan; The Volcanological Society of Japan; The Geodetic Society of Japan; The Japanese Society for Planetary Sciences; TERRAPUB region near the magnetic equator to the ground leads to an expectation that the onset times in the magnetometer data will lag the onset of pulsations in the riometer data by several minutes. An extensive examination of 7 years of simultaneous riometer and magnetometer data from South Pole station by Paquette et al. (1994) showed that only about a third of Pc4-5 events fit the predictions of Coroniti and Kennel, while in other events the onset of magnetic and precipitation pulsations was nearly simultaneous, and possible alternative generation mechanisms are to be explored.

Small-scale dispersive Alfvenic waves (DAW) can effectively accelerate and precipitate electrons due to their parallel electric field $E_{\|}$. However, this $E_{\|}$becomes substantial only in DAW with very small transverse scale comparable with the dispersive radius $\rho_{d}$, because $\left|E_{z}\right|=$ $\left(k_{\perp} \rho_{d}\right)^{2}\left(k_{z} / k_{\perp}\right)\left|E_{\perp}\right|$ (Borovsky, 1993; Stasiewicz et al., 2000). Rankin et al. (1999) estimated that the $E_{\|}$field that can occur in typical Alfvenic structures may cause additional acceleration by only about $100 \mathrm{eV}$, which is not sufficient for initiation of significant auroral optical emission or ionospheric plasma ionization. Therefore other mechanisms should be invoked.

An important feature of the auroral magnetosphereionosphere system is the occurrence of the auroral acceleration region (AAR) — a localized region with a substantial potential drop along the field lines. The AAR is assumed to produce an electron acceleration, necessary for auroral excitation. The AAR was recently shown to be an effective reflector of small-scale Alfven waves (Vogt and Haerendel, 1998; 


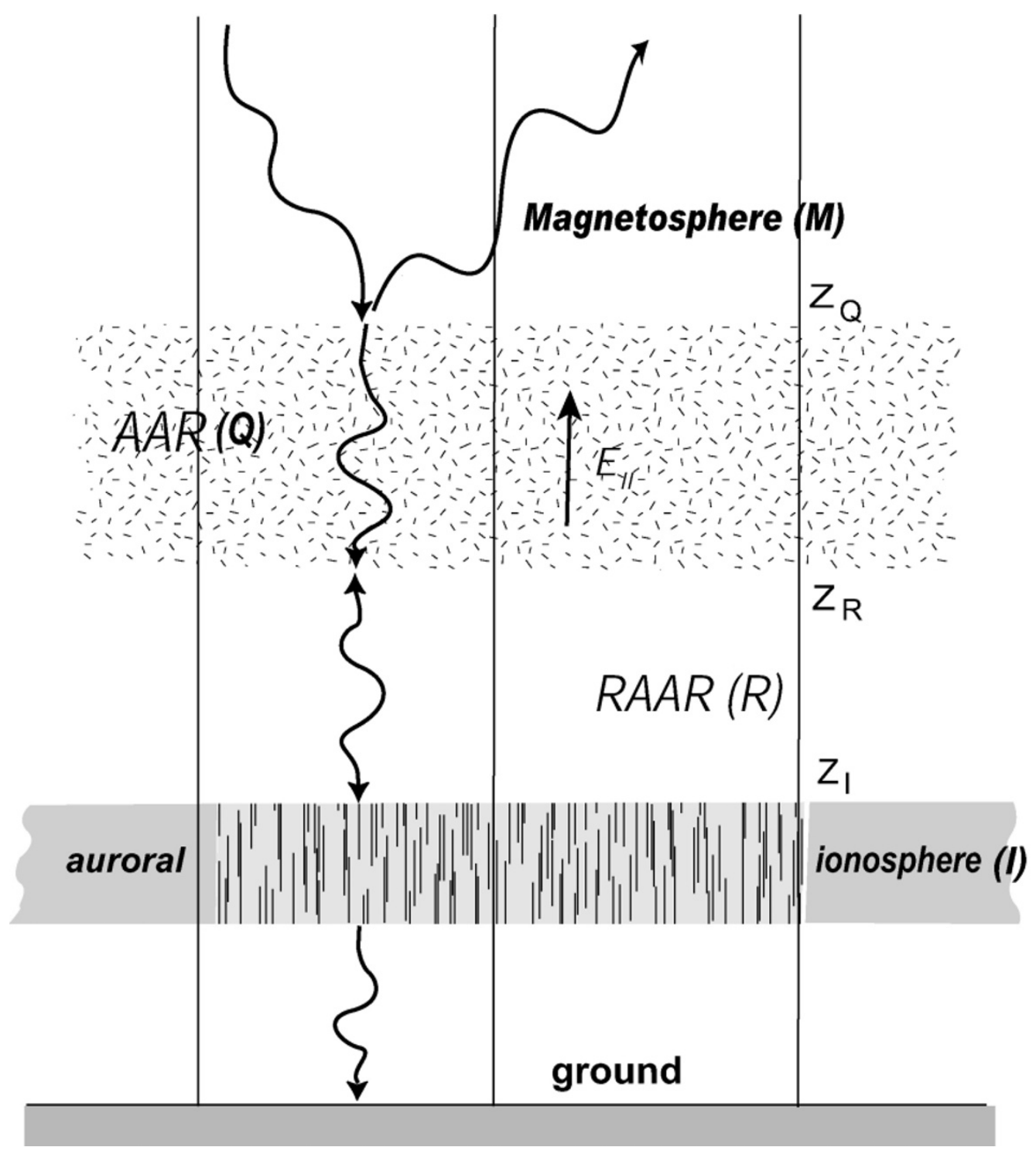

Fig. 1. A sketch of the model of the interaction of Alfven waves (shown by wavy lines) with the AAR in the topside ionosphere.

Fedorov et al., 2001). Thus, a resonator for Alfven waves can be formed between the bottom boundary of the AAR and the $E$-layer of the ionosphere (Pilipenko et al., 2002). This AAR-associated resonator (further named for brevity the RAAR) may be responsible for the trapping of smallscale Alfvenic structures in the topside auroral ionosphere, and formation of the ULF emission spectrum in the range around $0.1 \mathrm{~Hz}$. The spectral features in this frequency range, noted by Arnoldy et al. (1998), cannot be interpreted with the mechanism of the ionospheric Alfven resonator (IAR). The IAR is formed in the upper ionosphere due to a partial reflection of Alfven waves from steep gradient of Alfven velocity at altitude $\sim 10^{3} \mathrm{~km}$ and has typical frequencies $\sim 1 \mathrm{~Hz}$ (Lysak, 1988; Belyaev et al., 1990).

Modulation of the field-aligned potential drop during ULF Alfven wave interactions with the AAR may be another mechanism, not examined so far, for modulating electron acceleration/precipitation. Additionally, the occurrence of the RAAR can produce fine spectral features in magnetic/particle variations in the frequency range around the RAAR eigenfrequency. In this paper we consider the interaction of magnetospheric Alfven waves with the auroral topside ionosphere, comprising the AAR and an AAR-associated resonator, and estimate the efficiency of this mechanism in modulating electron acceleration/precipitation.

\section{Electrodynamic Model of the Auroral Region and Basic Equations}

A field-aligned potential drop immersed into a warm plasma is typically located in the auroral topside ionosphere at an altitude $\sim 1 R_{E}$, and this potential drop is concentrated within a layer with thickness of about a few thousands of $\mathrm{km}$ (Reiff et al., 1993), much less than the length of an auroral field line. From below, the topside ionosphere is bounded by a thin ionospheric E-layer. Magnetospheric Alfven waves impinge on the topside auroral ionosphere from above and may partially reflect back, be absorbed, excite the RAAR modes, and penetrate to the ground, as schematically shown in Fig. 1.

To describe theoretically these complicated wave patterns, we use a simplified multi-layer model of the auroral topside 
ionosphere (Fedorov et al., 2001; Pilipenko et al., 2002), assuming that the transition between layers is narrow as compared with the Alfven wave length. Each layer is labeled by index $m$, where $m=M$ refers to the magnetosphere above the AAR, $m=Q$ refers to the layer with a potential drop (AAR), $m=R$ refers to the RAAR, and $m=I$ refers to the $E$-layer of the ionosphere with the height-integrated conductivities $\Sigma_{P}$ and $\Sigma_{H}$. The plane $z=z_{I}$ corresponds to the $E$-layer of the ionosphere, the plane $z=z_{R}$ to the lower boundary of the AAR, and $z=z_{Q}$ to the upper boundary of the AAR. Above the AAR, $z \geq z_{Q}$, is the magnetosphere. The vertical scale of the AAR is $d_{Q}=z_{Q}-z_{R}$, and that of the AAR-associated resonator is $d_{R}=z_{R}-z_{I}$. The width of each layer is much less than the Alfven wave length, so it is reasonable in order to avoid unnecessary complications to replace in the theoretical model each realistic layer by a homogeneous layer with the altitude-averaged characteristics. Thus, each layer is assumed to be homogeneous with constant plasma density $N_{m}$, Alfven velocity $V_{A m}$, and Alfven wave conductance $\Sigma_{A m}=\left(\mu_{0} V_{A m}\right)^{-1}$. The neglected altitude dependence of the transverse cross-section of a flux tube would modify numerical estimates, but introduce no substantial modification of physical effects. Therefore, we use a simplified model with Cartesian geometry with the $x$ and $y$ axes parallel to the ionosphere, and the homogeneous straight geomagnetic field $\mathbf{B}_{0}=B_{0} \hat{\mathbf{z}}$ is along the vertical axis $z$.

A self-consistent kinetic distribution of particles and fieldaligned electric field in a mirror-confined plasma can be modeled, following Knight (1973), Vogt and Haerendel (1998), and Janhunen (1999), on the MHD level by the nonlocal current-voltage $(\mathrm{CV})$ relationship between the upward current $j_{\|}$and the potential drop $\Delta \Phi$ across the AAR as follows

$$
\Delta \Phi=-Q j_{\|}
$$

where $Q$ is the height-integrated resistance of the flux tube. Observations with sounding rockets and satellites have verified that the linearization of the full Knight formulation holds even for very high potential drops $\left(1-10^{2} \mathrm{kV}\right)$ and temperatures found above auroral arcs (Weimer et al., 1987; Lu et al., 1991) and in substorm onsets and westward-traveling surges (Olsson et al., 1996). The validity of the linear CV relationship (1) does not say anything about the origin of the potential drop, only its effect on precipitating particles. Such a relationship can arise from magnetic mirroring.

In the derivation of the full self-consistent $\mathrm{CV}$ relationship in a mirror-confined plasma (the "Knight formula") it was implicitly assumed that the potential and current across the AAR do not change in time considerably during the electron transit through the AAR, $t_{1} \simeq d_{Q} / V_{e}$, and time $t_{2} \simeq 2 d_{R} / V_{e}$ for the accelerated electrons to be mirrored back to the AAR. The transit time for $10-\mathrm{keV}$ electrons is estimated to be $t_{1} \sim$ $0.1 \mathrm{~s}$, whereas the mirroring time is $t_{2} \sim 1 \mathrm{~s}$. In general, temporal variations of the AAR with time scale $T$ may be considered as quasi-stationary when

$$
T \gg \max \left(t_{1}, t_{2}\right) \simeq 2 d_{R}(2 e \Delta \Phi / m)^{-1 / 2} .
$$

For low frequency ULF disturbances ( $\mathrm{Pc} 3-5 / \mathrm{Pi} 2$ range) the condition (2) is clearly valid, but in the high-frequency range
(Pc2/Pi1), comparable with the RAAR eigenfrequencies, this condition may become questionable. Nevertheless, as follows from (2) if the potential drop is larger than a certain, quite reasonable, value, namely $70 \mathrm{~V}$, the quasi-steady condition remains valid even for periods comparable with the RAAR eigen periods. Additionally it was supposed that the upward current is carried by magnetospheric electrons, undergoing adiabatic motions. The time scale of the ULF processes is long enough to guarantee the validity of the adiabatic approximation. This consideration justifies the use of the CV relationship in the form of a non-local Ohm's law (1) for the treatment of Alfven wave interactions with the AAR in other studies (e.g., Vogt et al., 1999; Kinney et al., 1999; Nakamura, 2000).

The parallel electric field $E_{z}$ in an Alfven wave can be produced by dispersive effects: electron inertia $\left(E_{z}=\mu_{0} \lambda_{e}^{2} \partial_{t} j_{z}\right.$, where $\lambda_{e}=c / \omega_{p}$ is the electron inertial scale, and $\omega_{p}$ is the plasma frequency) or the mirror force $\left(E_{z} \propto Q\right)$. Pilipenko et al. (2002) indicated that the mirror force dispersion dominates the electron inertia dispersion in the topside ionosphere for disturbances with transverse scales larger than $\lambda_{e} \simeq 0.1 \mathrm{~km}$. Therefore in what follows the electron inertial effects will be neglected to make the relationships simpler.

The spatial field-aligned distribution of potential $\Phi$ depends in a complicated way on the electron and ion distribution functions in the AAR. Here and further we consider the altitude-averaged value of an Alfven wave $E_{z}$. This field is assumed to be totally determined by the mirror resistance $Q$

$$
E_{z}=\left(Q / d_{Q}\right) j_{z}
$$

The relationship (3) does not imply that the AAR is just replaced by a resistor. In fact, the mirror force is nondissipative, and electrons transport the energy acquired inside the AAR to the ionosphere.

The electromagnetic Alfven-type disturbances in a laterally-homogeneous medium can be described by the vector potential $\mathbf{A}=A \hat{\mathbf{z}}$ and the scalar potential $\varphi$. Assuming a harmonic solution $\propto \exp \left(-i \omega t+i \mathbf{k}_{\perp} \mathbf{r}_{\perp}\right)$ we obtain for these wave potentials the following system of equations

$$
\begin{aligned}
\partial_{z} A-i\left(\omega / V_{A}^{2}\right) \varphi & =0 \\
{\left[-i \omega+\left(Q / \mu_{o} d_{Q}\right) k_{\perp}^{2}\right] A+\partial_{z} \varphi } & =0 .
\end{aligned}
$$

The field-aligned current $j_{z}$, and magnetic and electric fields $\mathbf{B}, \mathbf{E}$ in an Alfven wave are related to its potentials $\varphi$ and $\mathbf{A}$ as follows

$$
\begin{aligned}
\mathbf{B} & =-i\left(\hat{\mathbf{z}} \times \mathbf{k}_{\perp}\right) A & \mathbf{E}_{\perp}=-i \mathbf{k}_{\perp} \varphi \\
E_{z} & =-\partial_{z} \varphi+i \omega A & j_{z}=\mu_{0}^{-1} k_{\perp}^{2} A .
\end{aligned}
$$

From (5) it follows that a shear Alfven wave propagating along the geomagnetic field in the positive (negative) direction transports the current $j_{z}=\mp \Sigma_{A} k_{\perp}^{2} \varphi$.

At the interfaces between layers the wave potentials are to be continuous. At the upper boundary of the AAR $\left(z=z_{Q}\right)$ the radiation condition for Alfven waves can be adopted, $\varphi=V_{A M} A$. At the $E$-layer of the ionosphere $\left(z=z_{I}\right)$ the boundary condition for Alfven wave potentials holds $\varphi=-\left(\mu_{0} \Sigma_{P}\right)^{-1} A$. This boundary condition assumes that the reverse influence on Alfven waves of compressional 
modes excited in the Hall layer of the ionosphere can be neglected. This effect may produce additional Alfven wave dispersion (Yoshikawa and Itonaga, 1996; Pokhotelov et al., 2000) which is too small for our consideration.

For the multi-layer model considered here, as follows from (4), in each homogeneous layer the dispersion equation for the Alfven mode holds (Lysak and Dum, 1983)

$$
\left(k_{z}^{(m)}\right)^{2} \equiv k_{m}^{2}=k_{A m}^{2}\left(1+i \frac{\lambda_{A m}^{2} k_{\perp}^{2}}{k_{A m} d_{m}}\right)
$$

where $k_{A m}=\omega / V_{A m}$ is the Alfven wave number. The Alfven damping scale $\lambda_{A}$ in (6), introduced by Vogt and Haerendel (1998) and Fedorov et al. (2001), characterizes the interaction of a shear Alfven wave with a region with an electric potential drop and occurrence of wave dispersion related to the mirror force resistance. Physically, the parameter $\lambda_{A} \equiv \lambda_{A Q}=\left(Q \Sigma_{A Q}\right)^{1 / 2}$ is a scale at which the field-aligned specific resistance matches the Alfven wave impedance, $Z_{A}=\Sigma_{A}^{-1}$. It was accounted for in (6) that mirror force dispersion dominates the electron inertia dispersion, that is $\lambda_{A} \gg \lambda_{e}$. Further, we use the dimensionless wave vector $\bar{k}_{\perp}=k_{\perp} \lambda_{A}$.

Each $m$-th layer in the model under consideration is characterized by its characteristic impedance, $Z_{m}=Z_{A} \frac{k_{m}}{k_{A m}}$, so $Z_{M}=Z_{A M}=\Sigma_{A M}^{-1}, Z_{Q}=Z_{A Q} \frac{k_{Q}}{k_{A Q}}$, and $Z_{R}=$ $Z_{A R}$. In each layer the wave field can be presented as a sum of up-going $\propto \exp \left[i k_{m}\left(z-z_{m}\right)\right]$ and down-going $\propto \exp \left[-i k_{m}\left(z-z_{m}\right)\right]$ inhomogeneous plane waves. For the waves incident from a layer $m$ onto the boundary between the layers $m$ and $m^{\prime}$, the impedance condition holds $\mu_{0} \varphi\left(z_{m}\right)=$ $\mp Z_{m m^{\prime}} A\left(z_{m}\right)$, where $Z_{m m^{\prime}}$ is the input (surface) impedance, and the sign is $-(+)$ if the layer $m$ is above (below) the layer $m^{\prime}$. Further on, we denote the surface impedance of the ionosphere as $Z_{I}$, that is $Z_{R I} \equiv Z_{I}=\Sigma_{P}^{-1}$. Under the radiation condition the surface impedance for Alfven waves incident on the magnetosphere from inside the AAR coincides with the characteristic impedance of an Alfven wave in the magnetosphere, $Z_{Q M}=Z_{M}$.

In a multi-layer medium, the impedance $Z_{m^{\prime}}$ and input impedance $Z_{m m^{\prime}}$ determine the reflection coefficient $R_{m m^{\prime}}$ of a wave propagating from a layer $m$ to a layer $m^{\prime}$ as follows

$$
R_{m m^{\prime}}=\frac{Z_{m}-Z_{m m^{\prime}}}{Z_{m}+Z_{m m^{\prime}}}
$$

An input impedance $Z_{m m^{\prime}}$ above a multi-layered medium can be derived via the layer's characteristic impedances from standard waveguide theory or magnetotelluric sounding theory. This relationship for the model considered here can be found in Pilipenko et al. (2002).

\section{Reflection Properties of an Alfven Wave}

For better insight into the physics of Alfven wave interaction with the combined magnetosphere-AAR-RAARionosphere system, we consider first the features of the Alfven wave reflection from interfaces between media with different parameters. An excellent review of the Alfven wave reflection properties of the auroral current circuit is given by Vogt (2002).
As follows from (7) the reflection from the thin ionospheric $E$-layer is described by the coefficient

$$
R_{I}=\frac{\tilde{\Sigma}_{P}-1}{\tilde{\Sigma}_{P}+1}
$$

where $\tilde{\Sigma}_{P}=\Sigma_{P} / \Sigma_{A R}$ is the dimensionless Pedersen conductance. When $\tilde{\Sigma}_{P} \gg 1$, which is commonly the case in a sunlit or precipitation-modified ionosphere, the reflection of Alfven waves occurs as from a conductor, that is $R_{I} \rightarrow 1$. In this case the electric fields of down-coming and up-going waves tend to cancel each other, whereas magnetic components are summed up.

The input impedance of the combined AAR + magnetosphere system for Alfven waves incident on the AAR from below is to be determined from the general relationship for a multi-layered system (7). For common ULF wave parameters the "optical" thickness of the AAR is small, and the AAR can be considered mathematically as a thin layer for Alfven waves. This means that when

$$
k_{A} \ll \min \left\{1,\left(k_{\perp} \lambda_{A}\right)^{-2}\right\}
$$

phase change and amplitude damping upon the wave propagation across the layer may be neglected. When additionally the parameter $\delta=\left(\mu_{o} \omega d_{Q} / 2 Q\right)^{-1 / 2}$ is larger than the transverse wave length (formally $\delta$ looks like a "skin depth" scale, though the actual damping scale is different), that is

$$
1 \ll k_{\perp} \delta \ll\left(k_{A} d_{Q}\right)^{-1}
$$

the relationship for the input impedance of a system comprising a thin AAR can be simplified. In this approximation, valid for a wide range of AAR parameters for disturbances with transverse scales less than several tens of $\mathrm{km}$, the impedance of the $Q-M$ interface is reduced to

$$
Z_{R Q} \simeq Z_{M} \bar{k}_{\perp}^{2}+Z_{M}
$$

where the term $Z_{M} \bar{k}_{\perp}^{2}=k_{\perp}^{2} Q$ is the integrated resistance of the thin AAR. Then, the reflection coefficient from the $Q-M$ interface of an Alfven wave incident from the AAR simplifies and reduces to the following

$$
R_{R Q}=\frac{Z_{A R}-Z_{A M}-Z_{A Q} \bar{k}_{\perp}^{2}}{Z_{A R}+Z_{A M}+Z_{A Q} \bar{k}_{\perp}^{2}} .
$$

When mirror effects are neglected, the relationship (12) describes the partial reflection of Alfven waves from the $V_{A}(z)$ jumps at the interfaces between layers

$$
R_{R Q}=\frac{V_{A R}-V_{A M}}{V_{A R}+V_{A M}} .
$$

In the realistic topside ionosphere the altitude dependence of $V_{A}(z)$ can be rather inhomogeneous, causing an additional reflection of Alfven waves at steep gradients. However, to reveal more clearly the dominating effect in the auroral ionosphere - the wave reflection/absorption in the AAR, the altitude dependence of $V_{A}(z)$ will be neglected (this assumption will be considered in the Discussion section). Thus, to exclude this reflection and avoid unnecessary complications, 
here and further we suppose that $V_{A R}=V_{A Q}=V_{A M}$. Then (12) is further reduced to

$$
R_{R Q}=-\frac{\bar{k}_{\perp}^{2}}{2+\bar{k}_{\perp}^{2}}
$$

The relationship (14) shows that small-scale disturbances, $\bar{k}_{\perp}^{2}>1$, reflect from the AAR as from an insulator, $R_{R Q}<$ 0 . This means that the electric fields of downcoming and upgoing Alfven waves are summed up, whereas the magnetic components tend to cancel each other.

\section{Spectral Features of the AAR-associated Res- onator}

Alfven wave reflections from the $E$-layer and the bottom boundary of the AAR provide conditions for the occurrence of an AAR-associated resonator (RAAR) in the topside ionosphere. The eigenfrequency of the RAAR can be found from the dispersion equation $D\left(\bar{\omega}, \bar{k}_{\perp}^{2}, R_{I}, R_{R Q}\right)=0$ (Pilipenko et al., 2002), depending on $\bar{\omega}=\omega / \omega_{A}$, the dimensionless frequency, where $\omega_{A}=\pi V_{A R} / d_{R}$ is a characteristic frequency of the resonator. For a thin AAR, the reflection coefficient (12) is frequency-independent, and the eigenfrequencies $\omega_{n}$, damping rates $\gamma_{n}$, and the $Q$-factor of the $n$-th harmonic in the resonator can be easily found from the dispersion equation

$$
\begin{gathered}
\bar{\omega}_{n}=k_{A R} d_{R}=\frac{\omega_{n}}{\omega_{A}}= \begin{cases}n+\frac{1}{2} & \text { for } \tilde{\Sigma}_{P}>1 \\
n & \text { for } \tilde{\Sigma}_{P}<1\end{cases} \\
\frac{\gamma_{n}}{\omega_{A}}=\frac{1}{2 \pi} \log \left|\frac{\tilde{\Sigma}_{P}-1}{\tilde{\Sigma}_{P}+1} \cdot \frac{\bar{k}_{\perp}^{2}}{\bar{k}_{\perp}^{2}+2}\right|, \quad Q_{n}=\frac{\omega_{n}}{2\left|\gamma_{n}\right|}
\end{gathered}
$$

The quality of the resonator is determined by the wave dissipation rate in the ionosphere and by the reflection features of the AAR. Alfven disturbances in the resonator will experience severe damping for large scales, $\bar{k}_{\perp}<1$, but damping becomes much less significant for small scales, $\bar{k}_{\perp}>1$.

As (15) shows, for a highly conductive ionosphere $\left(\tilde{\Sigma}_{P}>\right.$ 1) the resonant oscillations are quarter-wave harmonics. Upon reflection from the AAR the phase of an Alfven wave changes by $180^{\circ}\left(R_{R Q}<0\right)$, whereas upon reflection from the ionosphere the phase does not change $\left(R_{I}>0\right)$. Under low-conductivity ionospheric conditions $\left(\tilde{\Sigma}_{P}<1\right)$ resonant oscillations are half-wave harmonics, because in this case the phase jump upon reflection from the AAR is still $180^{\circ}$, whereas reflection from the ionosphere produces an additional phase jump of $180^{\circ}\left(R_{I}<0\right)$.

For the AAR of finite thickness the spectral properties of the RAAR were found numerically by Pilipenko et al. (2002). The finite AAR width does not violate the main properties of the resonator, though somewhat reduces the reflection from the upper boundary and decreases the $Q$ factor.

\section{Interaction of Magnetospheric Alfven Waves with the AAR}

Here we consider different, but mutually related, aspects of the Alfven wave interaction with the auroral ionosphere:
1) backward reflection into the magnetosphere; 2) penetration inside the AAR and modulation of the field-aligned potential drop; 3) excitation of the AAR-associated resonator; and 4) the ground magnetic response. In the subsequent sections we combine the results for two distinct frequency ranges of ULF Alfven waves: low-frequency waves $(\omega \ll$ $\left.\omega_{A}\right)$ and high-frequency waves with frequencies comparable to the RAAR eigenfrequencies $\left(\omega \simeq \omega_{A}\right)$.

\subsection{Reflection of Alfven waves from the topside auroral ionosphere}

The interaction of a magnetospheric Alfven wave, $\propto$ $\exp \left[-i k_{A}\left(z-z_{Q}\right)\right]$, with the topside auroral ionosphere can be described with the help of the input impedances of the multi-layer system under consideration. The input impedance of the combined system AAR + RAAR + ionosphere can be found for the thin AAR using the same approach as Pilipenko et al. (2002)

$$
Z_{M Q}=Z_{A} \bar{k}_{\perp}^{2}+Z_{Q R}=Z_{A}\left(\bar{k}_{\perp}^{2}-1+\kappa^{2}(\bar{\omega})\right)
$$

where $Z_{A} \bar{k}_{\perp}^{2}=\Sigma_{Q}^{-1}$ is an integrated resistance of the thin AAR. The function

$$
\kappa^{2}(\bar{\omega})=2\left[1+R_{I} \exp (2 i \pi \bar{\omega})\right]^{-1}
$$

where $\operatorname{Re}(\kappa)>0$, accounts for the occurrence of the RAAR.

The above impedance $Z_{M Q}$ determines the coefficient of the magnetospheric Alfven wave reflection from the combined auroral system, that is

$$
R_{M Q}\left(\bar{\omega}, \bar{k}_{\perp}\right)=-1+\frac{2 Z_{A}}{Z_{A}+Z_{M Q}}=-1+\frac{2}{\bar{k}_{\perp}^{2}+\kappa^{2}(\bar{\omega})}
$$

The occurrence of the resonator beneath the AAR influences significantly the reflection of Alfven waves. For lowfrequency $\left(\omega \ll \omega_{A}\right)$ magnetospheric Alfven waves, as well as for waves with frequency matching the frequency of the RAAR integer harmonics, that is $\bar{\omega}=n(n=$ $0, \pm 1, \pm 2, \ldots)$, the coefficient of reflection (18) is reduced to the following

$$
R_{M Q}(n)=R_{M Q}^{(0)}=\frac{\tilde{\Sigma}_{P}-1-\tilde{\Sigma}_{P} \bar{k}_{\perp}^{2}}{\tilde{\Sigma}_{P}+1+\tilde{\Sigma}_{P} \bar{k}_{\perp}^{2}}
$$

The relationship (19) coincides with the one obtained and examined in detail by Vogt and Haerendel (1998) and Fedorov et al. (2001) for low-frequency Alfven waves, $\omega \ll \omega_{A}$, when the phase change inside the RAAR can be neglected.

For a magnetospheric Alfven wave with frequency matching the frequency of the semi-integer RAAR harmonics, $\bar{\omega}=n+1 / 2$, the relationship (18) reduces to

$$
R_{M Q}\left(n+\frac{1}{2}\right)=R_{M Q}^{(1)}=\frac{\tilde{\Sigma}_{P}^{-1}-1-\tilde{\Sigma}_{P}^{-1} \bar{k}_{\perp}^{2}}{\tilde{\Sigma}_{P}^{-1}+1+\tilde{\Sigma}_{P}^{-1} \bar{k}_{\perp}^{2}} .
$$

The difference between the relationships (19) and (20) is due to the fact than an Alfven wave acquires in the RAAR the additional phase shift multiple of $2 \pi$ in the first case, and multiple of $\pi$ in the second case.

The reflection coefficient $R_{M Q}$ depends on the transverse wave vector $\bar{k}_{\perp}$. The dependences of the absolute value (upper panel) and phase (lower panel) of $R_{M Q}\left(\bar{k}_{\perp}\right)$ are shown 

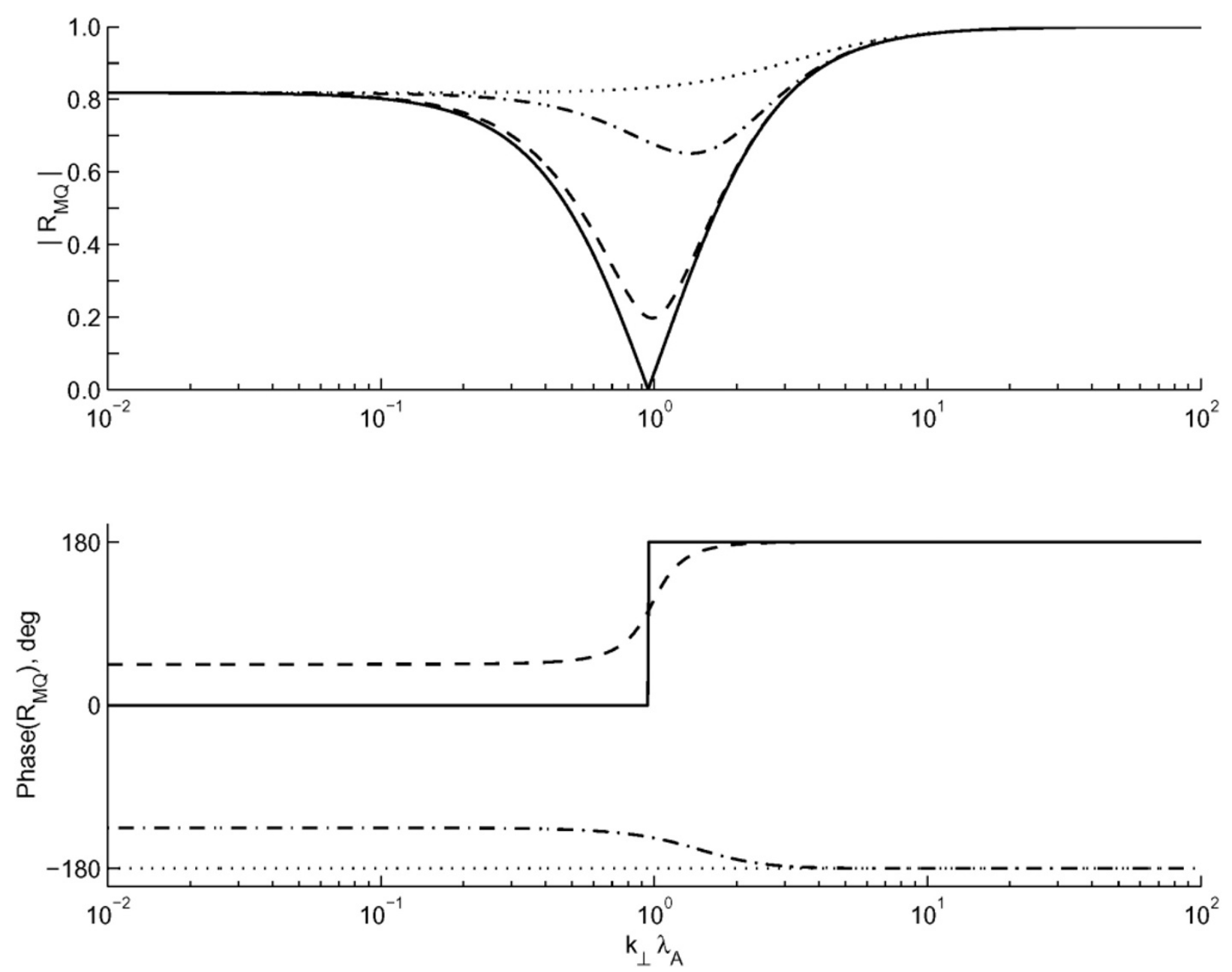

Fig. 2. The dependence of the absolute value (upper panel) and phase (lower panel) of the reflection coefficient $R_{M Q}$ for $\tilde{\Sigma}_{P}=10$ on the dimensionless transverse wave vector $\bar{k}_{\perp}$ for the following fixed frequencies: $\omega=n \omega_{A}$, (solid), $\omega=(n+1 / 8) \omega_{A}($ dashed $)$, and $\omega=(n+1 / 2) \omega_{A}($ dotted), where $n=0, \pm 1, \pm 2, \ldots$. The dashed-dotted line corresponds to $\tilde{\Sigma}_{P}=0.1$, and $\omega=(n+1 / 8) \omega_{A}$.

in Fig. 2 for several fixed frequencies: $\omega=n \omega_{A}$ (solid line), $\omega=(n+1 / 8) \omega_{A}$ (dashed), and $\omega=(n+1 / 2) \omega_{A}$ (dotted), where $n=0, \pm 1, \pm 2, \ldots ; \tilde{\Sigma}_{P}=10$. The dasheddotted line corresponds to an ionosphere with low conductivity $\left(\tilde{\Sigma}_{P}=0.1\right)$, and $\omega=(n+1 / 8) \omega_{A}$. According to this plot, Alfven waves are effectively reflected back $\left(\left|R_{M Q}\right| \simeq 1\right)$ into the magnetosphere from the combined AAR + RAAR + ionosphere system for all the transverse wave scales except for a range around $\lambda_{A}$.

However, the phases of the reflected waves are different for small and large scales. For a highly conductive ionosphere, when $\tilde{\Sigma}_{P}>1$, the phase of the reflection coefficient jumps from 0 to $180^{\circ}$ at $\bar{k}_{\perp}=1$, whereas for $\tilde{\Sigma}_{P}<1$, the phase of $R_{M Q}$ is $-180^{\circ}$ for all wave vectors. This phase behavior is due to the fact that the reflection of magnetospheric Alfven waves with large scales occurs predominantly from the ionospheric $E$-layer, whereas reflection of waves with small scales does so from the AAR.

Other properties of the reflection coefficient $R_{M Q}$ are summarized in the Appendix. From these properties (A1) it follows that the dotted line in Fig. 2 also corresponds to the case $\omega=n \omega_{A}$, and the solid line corresponds also to $\omega=(n+1 / 2) \omega_{A}$, but for the parameter $\tilde{\Sigma}_{P}=0.1$.

The dependence of the reflection coefficient $R_{M Q}$ on fre- quency in the band around $\omega_{A}$ has an oscillatory character, but the depth of the modulation depends on the wave scales. Examination of analytical properties of the function in the right-hand part of (18) in the Appendix shows that the magnitude of $R_{M Q}$ varies between extreme values $\left|R_{M Q}^{(0)}\right|$ and $\left|R_{M Q}^{(1)}\right|$, which are reached at frequencies $\bar{\omega}=n$ and $\bar{\omega}=n+1 / 2$, respectively. Figure 3 shows the frequency dependences of the modulus and phase of $R_{M Q}$ for a highly conductive ionosphere, $\tilde{\Sigma}_{P}=10$. The solid line corresponds to $\bar{k}_{\perp}=\left(1-1 / \tilde{\Sigma}_{P}\right)^{1 / 2}$, when the frequency modulation is greatest, and the dashed line corresponds to $\bar{k}_{\perp}=2$. As is evident from Fig. 3(a), the reflection of the magnetospheric Alfven wave from the topside auroral ionosphere is highest at frequencies $\omega_{n}=\omega_{A}(n+1 / 2)$, which coincide with the RAAR resonant frequencies (15). The reflection is lowest at the "semi-resonant" frequencies

$$
\bar{\omega}=\frac{\bar{\omega}_{n}+\bar{\omega}_{n+1}}{2}=\left\{\begin{array}{cc}
n & \text { for } \tilde{\Sigma}_{P}<1 \\
n+\frac{1}{2} & \text { for } \tilde{\Sigma}_{P}>1
\end{array} .\right.
$$

The effectively reflected waves are nearly in-phase with incident waves (Fig. 3(b)). 

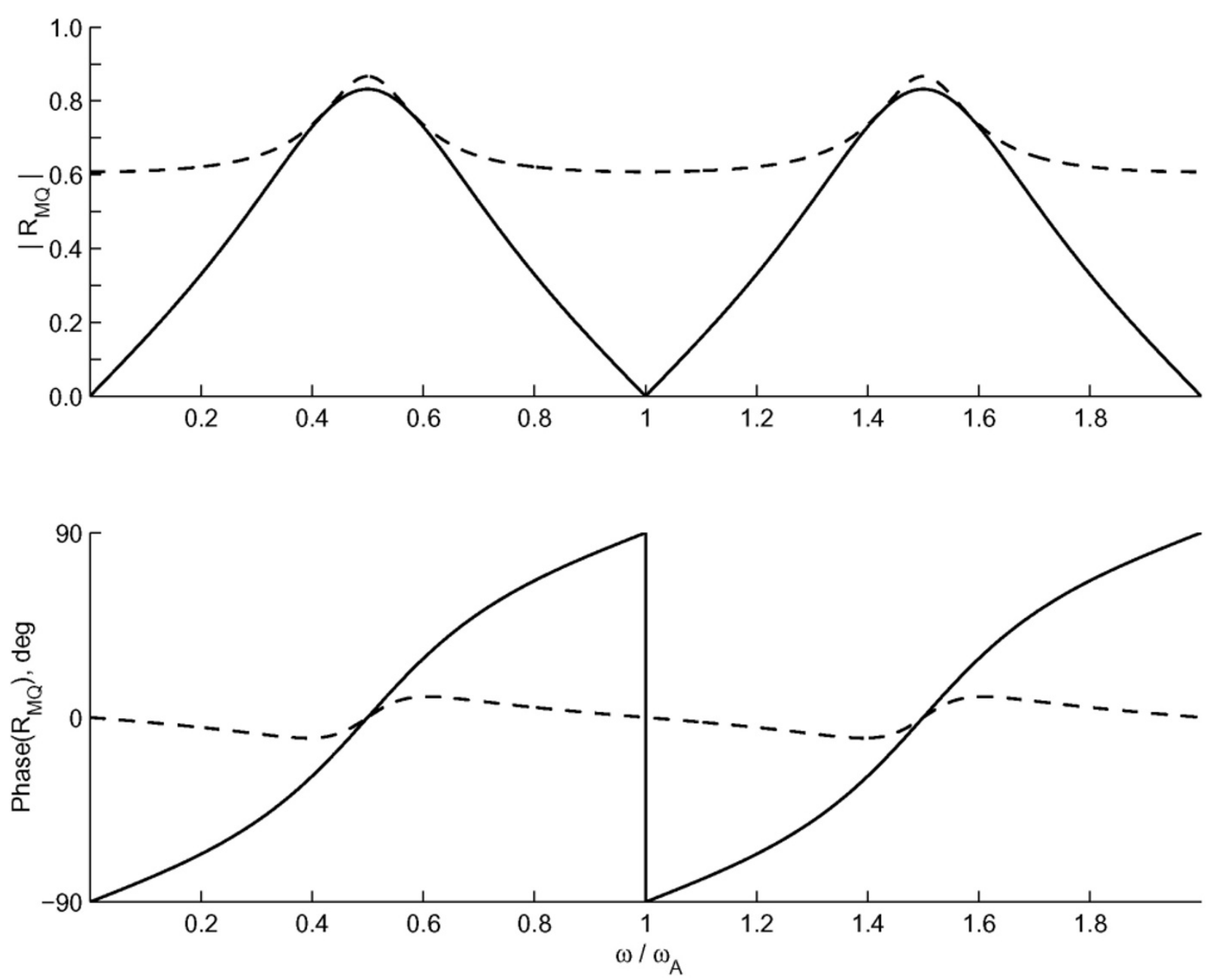

Fig. 3. The frequency dependence of the modulus (upper panel) and phase (lower panel) of the reflection coefficient $R_{M Q}$ at $\tilde{\Sigma}_{P}=10$ for the critical wave vector $\bar{k}_{\perp}=\bar{k}_{*}$ (solid), and $\bar{k}_{\perp}=2$ (dashed) on the dimensionless frequency $\omega / \omega_{A}$.

\subsection{Modulation of the potential drop by magneto-} spheric Alfven waves

The reflection coefficient $R_{M Q}$ determines the part of the oscillatory field-aligned current, $j_{z}\left(z_{Q}\right)$, transported by magnetospheric Alfven waves, which penetrates into the AAR

$$
j_{z}\left(z_{Q}\right)=j_{z M}^{(i)}\left(1+R_{M Q}\right) .
$$

Here the factor $1+R_{M Q}\left(\bar{\omega}, \bar{k}_{\perp}\right)$ is determined by (18), and $j_{z M}^{(i)}$ is the amplitude of field-aligned current in an Alfven wave incident from the magnetosphere.

The magnetospheric Alfven wave penetrating inside the AAR induces oscillations of the field-aligned potential drop $\Delta \Phi$. Variations of the potential drop $\Delta \Phi$ by an Alfven wave can be found by multiplying the magnitude of the oscillatory current (22) by the AAR resistance $Q$. Thus, the amplitude of these oscillations normalized to the amplitude of the incident Alfven wave potential $\varphi_{M}^{(i)}$ is as follows

$$
\frac{\Delta \Phi}{\varphi_{M}^{(i)}}=-\frac{Q j_{z}\left(z_{Q}\right)}{\varphi_{M}^{(i)}} .
$$

We introduce the modulation factor, normalized to the amplitude of a magnetospheric wave magnetic component $B_{M}^{(i)}=$ $i k_{\perp} \mu_{0} \Sigma_{A} \varphi_{M}^{(i)}$, and derive it via the dimensionless coefficient of the potential modulation $T_{\Phi}$. From $(22,23)$ it follows that

$$
\begin{aligned}
\frac{\Delta \Phi}{B_{M}^{(i)}} & =K_{\Phi} T_{\Phi} \\
T_{\Phi}\left(\bar{\omega}, \bar{k}_{\perp}\right) & =-i \bar{k}_{\perp}\left(1+R_{M Q}\right)=-\frac{2 i \bar{k}_{\perp}}{\bar{k}_{\perp}^{2}+\kappa^{2}(\bar{\omega})} .
\end{aligned}
$$

The coefficient $K_{\Phi}=\mu_{0}^{-1}\left(Q / \Sigma_{A}\right)^{1 / 2}$ can be visualized as the amplitude of the oscillating potential when all the field-aligned current transported by an incident Alfven wave with unit amplitude and transverse scale matching the Alfven damping scale $\lambda_{A}$ would penetrate into the AAR.

For low-frequency ULF waves $\left(\omega \ll \omega_{A}\right)$ the amplitude of the potential drop modulation as follows from (17) and (24) is

$$
\frac{\Delta \Phi}{B_{M}^{(i)}}=-i K_{\Phi} \frac{2 \bar{k}_{\perp}}{1+\tilde{\Sigma}_{P}^{-1}+\bar{k}_{\perp}^{2}} .
$$

For low-frequency waves the induced oscillations of the potential drop are largest at the transverse scale $\bar{k}_{*}=$ $\left(1+\tilde{\Sigma}_{P}^{-1}\right)^{1 / 2}$ and are as follows:

$$
\left|\frac{\Delta \Phi}{B_{M}^{(i)}}\right|_{\bar{k}_{\perp}=\bar{k}_{*}}=\frac{K_{\Phi}}{\left(1+\tilde{\Sigma}_{P}^{-1}\right)^{1 / 2}} .
$$



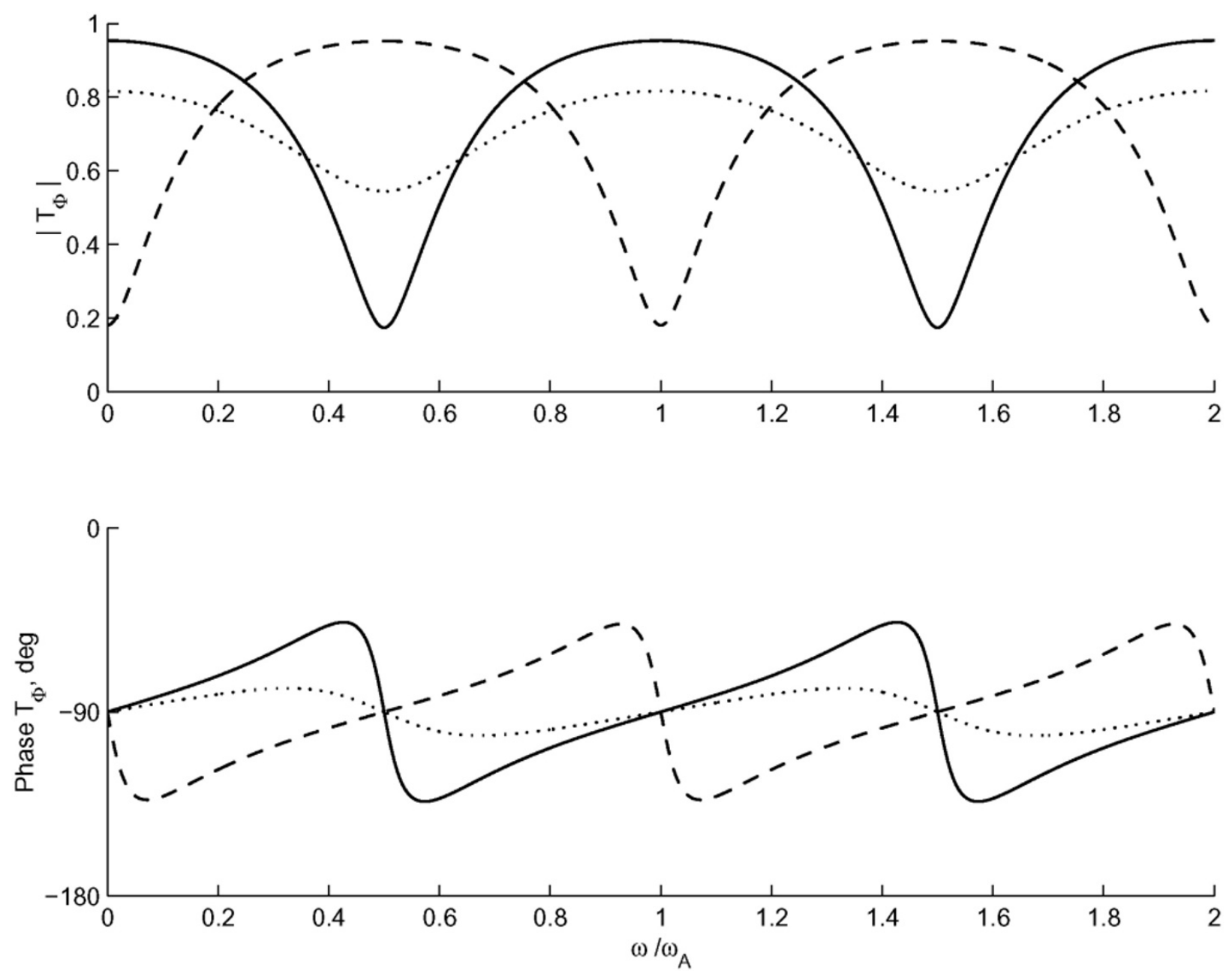

Fig. 4. The frequency dependence of the absolute value (upper panel) and phase (lower panel) of the dimensionless excitation coefficient $T_{\Phi}(\bar{\omega})$ for selected values of the ionospheric Pedersen conductivity $\tilde{\Sigma}_{P}: 10$ (solid line) 2 (dotted line), and 0.1 (dashed line) for $\bar{k}_{\perp}=\bar{k}_{*}$.

The oscillation amplitudes grow upon increase of the ionospheric Pedersen conductance and reach maximal value $K_{\Phi}$ at $\tilde{\Sigma}_{P} \rightarrow \infty$.

The properties of the dimensionless coefficient $T_{\Phi}$ of the potential drop oscillation excitation are examined in the Appendix. This coefficient $T_{\Phi}(\bar{\omega})$ in the frequency range around $\omega_{A}$ has an oscillatory dependence on frequency. It reaches maximum values

$$
\max \left|T_{\Phi}(\bar{\omega})\right|=\left\{\begin{array}{l}
\rho_{2} \text { for } \tilde{\Sigma}_{P}>1 \\
\rho_{1} \text { for } \tilde{\Sigma}_{P}<1
\end{array}\right.
$$

at "mid-resonant" frequencies $\left(\bar{\omega}_{n}+\bar{\omega}_{n+1}\right) / 2(21)$, where $\rho_{1}$ and $\rho_{2}$ are determined by (A2). At resonant frequencies $\bar{\omega}_{n}$ (15) the coefficient $\left|T_{\Phi}\right|$ has minimal values:

$$
\min \left|T_{\Phi}(\bar{\omega})\right|=\left\{\begin{array}{l}
\rho_{1} \text { for } \tilde{\Sigma}_{P}>1 \\
\rho_{2} \text { for } \tilde{\Sigma}_{P}<1
\end{array} .\right.
$$

At $\Sigma_{P} \rightarrow 0$ or $\rightarrow \infty$ the minimal and maximal values of $\left|T_{\Phi}\right|$ are 0 and 1 , respectively.

The relationship (A2) shows that the efficiency of the potential drop modulation by an Alfven wave depends on its transverse scale. Modulation is highest at "mid-resonant" frequencies when the transverse wave vector $\bar{k}_{\perp}=\bar{k}_{*}$, where

$$
\bar{k}_{*}=\left\{\begin{array}{ll}
\left(1+\tilde{\Sigma}_{P}^{-1}\right)^{1 / 2} & \text { for } \tilde{\Sigma}_{P}>1 \\
\left(1+\tilde{\Sigma}_{P}\right)^{1 / 2} & \text { for } \tilde{\Sigma}_{P}<1
\end{array} .\right.
$$

These regularities are illustrated by Fig. 4, which shows the frequency dependence of the absolute values (upper panel) and phase (lower panel) of the dimensionless modulation coefficient $T_{\Phi}(\bar{\omega})$ at $\bar{k}_{\perp}=\bar{k}_{*}$ for 3 selected values of $\tilde{\Sigma}_{P}$ : 10 (solid line), 2 (dotted line), and 0.1 (dashed line). The most prominent oscillations of the potential drop are excited in frequency bands between the RAAR resonant frequencies for a given conductance, whereas at resonant frequencies oscillations of $\Delta \Phi$ are minimal.

\subsection{Excitation of the RAAR modes}

An incident Alfven wave burst with a wide spectrum, penetrating through the AAR, can further excite the RAAR modes and penetrate through the $E$-layer to the ground. Let us estimate the amplitude of oscillations generated inside the AAR-associated resonator and on the ground by an Alfven wave incident from the magnetosphere. The structure of an Alfven wave in the resonator can be presented as follows

$$
B_{R}(z)=B_{R}^{(i)}\left\{\exp \left[-i k_{A}\left(z-z_{I}\right)\right]+R_{I} \exp \left[i k_{A}\left(z-z_{I}\right)\right]\right\}
$$



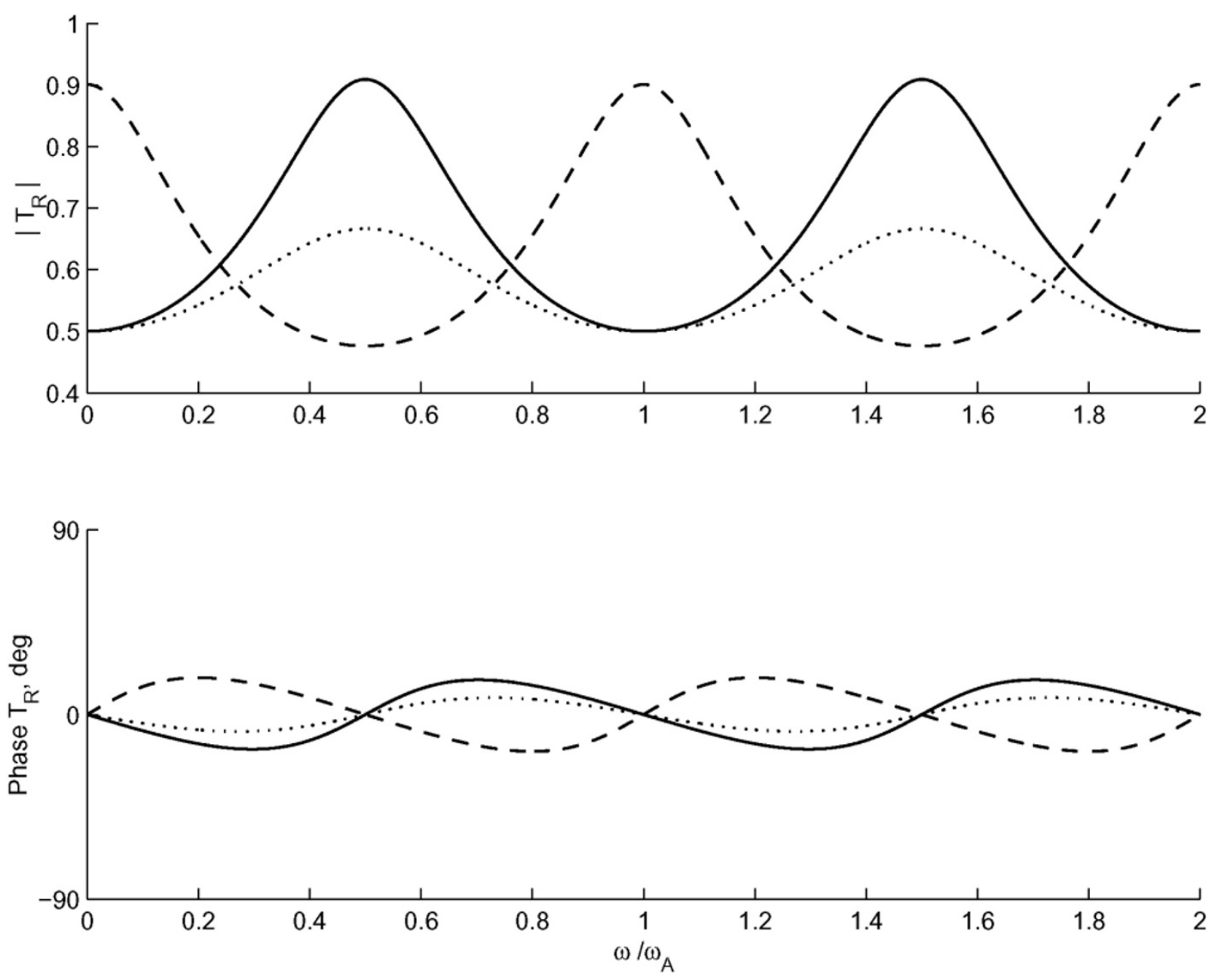

Fig. 5. The frequency dependence of the transmission coefficient $T_{R}(\bar{\omega})$ for the same values of $\bar{k}_{\perp}$ and $\tilde{\Sigma}_{P}$ as in Fig. 4 .

where $B_{R}^{(i)}$ is the amplitude of the Alfven wave incident on the lower boundary of the resonator ( $E$-layer) from the RAAR.

We introduce the coefficient $T_{R}$ of the resonator excitation as the ratio of the amplitude of the wave incident on the ionosphere, $B_{R}^{(i)} \exp (-i \pi \bar{\omega})$, to the amplitude of an Alfven wave $B_{M}^{(i)}$, incident from the magnetosphere upon the AAR. The factor $\exp (-i \pi \bar{\omega})=\exp \left(-i k_{A R} d_{R}\right)$ has been introduced to account for the phase increase upon the Alfven wave propagation from the AAR towards the ionosphere. As a result, we obtain

$$
T_{R}\left(\bar{\omega}, \bar{k}_{\perp}\right)=\frac{B_{R}^{(i)} \exp (-i \pi \bar{\omega})}{B_{M}^{(i)}}=\frac{1+R_{M Q}}{1+R_{I} \exp (2 i \pi \bar{\omega})}
$$

Substituting $1+R_{M Q}$ from (18) into (30), the coefficient $T_{R}$ can be expressed as follows

$$
T_{R}=\frac{\kappa^{2}}{\bar{k}_{\perp}^{2}+\kappa^{2}} .
$$

If the AAR resistance is neglected, that is $Q=0$, then in (31) $\bar{k}_{\perp}=0$ and evidently $T_{R}=1$.

The frequency dependence of $T_{R}(\bar{\omega})$ is shown in Fig. 5 for the same values of $\bar{k}_{\perp}$ and $\tilde{\Sigma}_{P}$ as in Fig. 4 . In contrast to the $T_{\Phi}(\bar{\omega})$ frequency dependence, the coefficient $T_{R}(\bar{\omega})$ achieves maximal values at resonant frequencies of the RAAR and minimal values at "mid-resonant" frequencies.

\subsection{Penetration of magnetospheric Alfven waves to the ground}

Now we estimate the expected frequency-dependence of the magnetic signal observed on the ground. This signal is produced by the height-integrated current induced in the $E$ layer $\left(z=z_{I}\right)$ by the electric field, $\mathbf{E}$, of an Alfven wave: $\mathbf{I}=\hat{\Sigma} \mathbf{E}$ (here $\hat{\Sigma}$ is the $2 \mathrm{D}$ tensor of the ionospheric heightintegrated conductivity). This current can be derived via the amplitude of the magnetic component $B_{M}^{(i)}$ of the incident Alfven wave and coefficient $T_{R}(30)$ as follows

$$
\mathbf{I}=\frac{2 \mu_{0}^{-1}}{\tilde{\Sigma}_{P}+1}\left(\tilde{\Sigma}_{P} \frac{\mathbf{k}_{\perp}}{\left|k_{\perp}\right|}+\tilde{\Sigma}_{H} \frac{\hat{\mathbf{z}} \times \mathbf{k}_{\perp}}{\left|k_{\perp}\right|}\right) T_{R} \exp (i \pi \bar{\omega}) B_{M}^{(i)}
$$

The ground magnetic field $\mathbf{B}_{g}$ is produced by the Hall part $\mathbf{I}_{H}$ of the ionospheric current (the second term in the righthand part of (32)). For an ideally conducting Earth, it can be estimated from the following relation

$$
\mathbf{B}_{g}=\mu_{0} \hat{\mathbf{z}} \times \mathbf{I}_{H} \exp \left(-k_{\perp} z_{I}\right) .
$$

Substituting $\mathbf{I}_{H}$ in the latter relationship we find

$$
\mathbf{B}_{g}=-\frac{2 \tilde{\Sigma}_{H} \exp \left(-k_{\perp} z_{I}\right)}{\tilde{\Sigma}_{P}+1} \frac{\mathbf{k}_{\perp}}{\left|\mathbf{k}_{\perp}\right|} T_{R} \exp (i \pi \bar{\omega}) B_{M}^{(i)} .
$$


This expression (33) coincides with known formulae for the ground magnetic field given by Hughes and Southwood (1976) and Pilipenko et al. (2000) if the AAR is absent, $Q=0$ and $T_{R}=1$, and the amplitude of the incident Alfven wave $B_{M}^{(i)}$ would be replaced by the total amplitude of the magnetic field above the ionosphere $B_{M}\left(z=z_{I}\right)$ according to $B_{M}^{(i)} \exp (i \pi \bar{\omega})=B_{M}\left(\tilde{\Sigma}_{P}+1\right) / 2 \tilde{\Sigma}_{P}$. Thus, the relationship (33) shows that the frequency dependence of the magnetic variations penetrating to the ground is determined by the coefficient $T_{R}(\omega)$ (Fig. 5).

For comparison with ground observations it is interesting to estimate the expected ratio between an additional ionospheric ionization $\Delta J / J \simeq \Delta \Phi / \Phi$ and a ground magnetic signal. For that it is important to know the relationship between the coefficients $T_{R}$ and $T_{\Phi}$. From equations (31) and (24) it follows that they are related as follows

$$
\frac{T_{\Phi}}{T_{R}}=-i \bar{k}_{\perp}\left[1+R_{I} \exp (2 i \pi \bar{\omega})\right]
$$

At low frequencies $\left(\omega \ll \omega_{A}\right)$ a relation between the modulation depth and ground magnetic signal is determined by the following relationship resulting from $(33,24)$ :

$$
\left|\frac{\Delta \Phi}{B_{g}}\right|=\bar{k}_{\perp} K_{\Phi} \frac{\tilde{\Sigma}_{P}+1}{2 \tilde{\Sigma}_{H}} \exp \left(k_{\perp} z_{I}\right)\left[1+R_{I} \exp (2 i \pi \bar{\omega})\right] .
$$

\section{Ground Magnetic Response to Modulated Pre- cipitation}

Alfven wave modulation of the electron acceleration in the auroral region will cause periodic variations of precipitating electron fluxes. Relationships between the variations of electron energy and variations of the electron ionization rate and conductance of the ionosphere can be reliably found only with the help of a numerical ionospheric model. Here we make simple estimates valid only within an order of magnitude.

We suppose that variations of the field-aligned current in the AAR, $j_{z}(t)=Q^{-1} \Delta \Phi$, modulate the ionospheric density, conductances, and currents, and, thus, can be a generation mechanism for an additional ground magnetic signal. Small variations of the plasma density in the ionosphere, that is $N=N_{e}+n(t)$, can be described with the linearized balance equation

$$
\partial_{t} n=q j_{z}-n / \tau
$$

where $q=\gamma / e \Delta z$ is the ionization source, derived via the ion production rate $\gamma$, and the thickness of the ionospheric conductive layer $\Delta z$. The recombination time $\tau$ in (36) is related to the recombination coefficient $\alpha_{R}$ and the ionospheric electron density $N_{e}$ at the altitude of the absorption layer as follows: $\tau^{-1}=2 \alpha_{R} N_{e}$. This time constant varies in the range 10-100 s, depending on the characteristic energy of precipitating electrons and altitude of the absorption layer (Rees, 1963). Periodic oscillations of $j_{z}(t)$ produce periodic plasma density variations in the $E$-layer ionosphere with amplitude

$$
n(t)=\frac{\gamma j_{z}(t)}{e \Delta z\left(-i \omega+\tau^{-1}\right)} .
$$

Let us assume that ground magnetic signals are produced only by the modulation of the ionospheric current by periodic variations of electron fluxes. The ionospheric electric field $\mathbf{E}_{0}$ inside a disturbed area may be somewhat decreased by the polarization electric field $\mathbf{E}^{\prime}$, that is $\mathbf{E}=\mathbf{E}_{0}-\mathbf{E}^{\prime}$. The exact calculations of this polarization decrease can be found elsewhere (e.g., Grant and Burns, 1995). As an order of magnitude approximation, the ground magnetic response $B_{g}$ to the periodic variations of ionospheric conductance $\Delta \Sigma_{H}$ with the electric field $\mathbf{E}$ can be estimated as $B^{(g)} \simeq \mu_{0} \Delta \Sigma_{H} E$. Taking into account that variations of the ionospheric conductance are caused by additional ionization by precipitating electrons, that is $\Delta \Sigma_{H} / \Sigma_{H} \simeq n / N, \Sigma_{H} \simeq \sigma_{H} \Delta z$, and the local conductivity in the $E$-layer $\sigma_{H} \simeq e N / B$, we arrive at

$$
B_{g}^{(\Sigma)} \simeq \mu_{0} \frac{\gamma V_{E}}{\left(-i \omega+\tau^{-1}\right)} j_{z}
$$

Here $V_{E}=E / B$ is the convection velocity in the ionosphere produced by electric field $\mathbf{E}$.

Now let us assume that the field-aligned current transported by Alfven waves is due to variations of the flux of precipitating energetic particles. The ground response to this current can be estimated from (33). Estimating the magnetic component of an Alfven wave with the transverse scale $l \simeq k_{\perp}^{-1}$ as $B^{(i)} \simeq \mu_{0} j_{z} k_{\perp}^{-1}$, and assuming that $\Sigma_{H} / \Sigma_{P} \simeq 1$, and $k_{\perp} z_{I}<1$, we arrive at

$$
B_{g}^{(A W)} \simeq \mu_{0} l j_{z} .
$$

In the low-frequency range $\omega \ll \tau^{-1}$, the comparison of these two mechanisms, described by (38) and (39), gives that

$$
\frac{B_{g}^{(\Sigma)}}{B_{g}^{(A W)}} \simeq \frac{\gamma V_{E} \tau}{l}
$$

For average parameters, $\tau \simeq 10 \mathrm{~s}, \gamma \simeq 10^{2}, l \simeq 2 \times$ $10^{2} \mathrm{~km}$, and $V_{E} \simeq 10^{2} \mathrm{~m} / \mathrm{s}$, the ratio (40) is about unity, indicating that both mechanisms may give comparable input to a ground magnetic signal.

This consideration shows that periodic precipitation produced by an Alfven wave cannot substantially increase the total ground response, even for values of $\gamma$ as high as $10^{2}$. However, depending on actual parameters in different situations this relationship may vary noticeably, especially in the range around resonant frequencies of the RAAR.

\section{Discussion}

In this paper a new mechanism of modulation of electron acceleration by Alfven waves has been proposed, which could be identified in riometer observations. This mechanism is closely associated with the occurrence of the AAR and can operate at auroral latitudes only.

We estimate characteristic parameters of the model considered. Typical values of the field-aligned resistance $Q$ are about $10^{7}-10^{9} \Omega \cdot \mathrm{m}^{2}$. For this value of resistance a field-aligned potential drop $\Delta \Phi \simeq 0.01-10 \mathrm{kV}$ is necessary to support the current $j_{\|} \sim 10^{-6}-10^{-5} \mathrm{~A} / \mathrm{m}^{2}$. Assuming that in the auroral topside ionosphere the Alfven velocity is $V_{A} \simeq 5 \times 10^{3} \mathrm{~km} / \mathrm{s}$, the corresponding Alfven wave conductance is $\Sigma_{A} \simeq 0.2 \Omega^{-1}$. The height-integrated Pedersen conductance due to the diffusive auroral precipitation varies in the range $\Sigma_{P} \simeq 1-10 \Omega^{-1}$. Therefore, the condition of a highly conductive ionosphere, $\tilde{\Sigma}_{P}>1$ prevails. 
It was also assumed that the non-local CV relationship (1) remains valid for a system on ULF time scales. The quasi-steady condition (2) is amply satisfied on the time scales corresponding to low-frequency ULF waves (Pc3-5 and $\mathrm{Pi} 2$ range), but it is near the quasi-stationary limit in the high-frequency (Pc2/Pi1) range. As commonly assumed (e.g. Wright et al., 2002) the predominant portion of the field-aligned current is carried by electrons accelerated in the AAR. We also assume that possible deviations $\Delta \Phi$ and $j_{\|}$ from their background values $\Delta \Phi^{(0)}$ and $j_{\|}^{(0)}$ do not shift the $\mathrm{CV}$ relationship (1) from its linear range.

The realistic finite-width AAR may be considered mathematically as a thin layer when the wavelength and "skindepth" of the Alfven wave are much larger than the layer width. In our formalism the AAR has been considered as a thin layer, whereas variations of fields and currents within the layer have been neglected. The relevant condition of this approximation is given by $(9,10)$. For a mean value of fieldaligned resistance $\sim 10^{8} \Omega \cdot \mathrm{m}^{2}$ and wave period $T \simeq 100 \mathrm{~s}$ the corresponding value of $\delta \sim 7 \mathrm{~km}$. Thus, the approximation of a thin AAR is valid for wave disturbances with transverse scales about a few tens of $\mathrm{km}$ and less, and the finite-width AAR indeed may be replaced by a thin layer.

Concurrent variations of ULF pulsations and cosmic noise absorption (CNA) are a ubiquitous element of magnetosphere-ionosphere interactions at auroral latitudes (Olson et al., 1980). These concurrent pulsations embrace an interval of periods from Pi1 $(\sim 10 \mathrm{~s})$ to $\mathrm{Pc} 5$ and Pi2 $(\sim$ several minutes). Pi1 band limited emissions are observed in the night-morning sector of the auroral oval and near noon at latitudes of the dayside cleft (Engebretson et al., 1983, 1984, 1986; Borovkova et al., 1992). The generation mechanism of morning Pilc is probably related to the precipitation of energetic electrons within the Region 2 currents, whereas for daytime Pi1c it is in the region of cleft-related field-aligned currents. The intensity of dayside broad-band Pc5 waves also was shown to correlate with precipitation of energetic particles at cusp/cleft latitudes. Moreover, quantitative estimates based on riometer and photometer observations indicate that variations in electron precipitation are sufficient to drive the ground broadband pulsations (Posch et al., 1999).

The extensive examination of simultaneous broad beam riometer, magnetometer and VLF data from South Pole station by Paquette et al. (1994) revealed nearly 200 pulsation events with long periods (100-1000 s) with consistent frequency in both electron precipitation and ground geomagnetic field variations. However, only about a third of these fit the predictions of the Coroniti and Kennel (1970) model: a disparity in onset times, together with modulation of an elevated level of VLF emissions. In other events the onset of magnetic and precipitation pulsations was nearly simultaneous, and the levels of VLF activity were not higher than those of a randomly selected test set. It may be suggested that these simultaneous events are probably attributable to the mechanism of AAR modulation.

If we assume that riometric variations $\Delta R \propto \Delta \Phi$, from (35) it follows that the ratio between the riometer signal and the ground magnetic signal in the Pc3-5 and Pi2 bands weakly depends on the ionospheric conductance, but is very sensitive to the transverse scale of the disturbance. The mod- ulation of the potential drop is most significant for wave scales of about the Alfven damping scale, $\lambda_{A}$, which is $\sim 10$ $\mathrm{km}$. From (33) it follows that spatially periodic waves with these scales will be nearly totally screened from the ground, e.g., for $k_{\perp} \simeq 10^{-1} \mathrm{~km}^{-1}$ the ground magnetic field will be decreased $2 \times 10^{4}$ times as compared with the ionospheric field. However, the situation may be different for a spatially localized Alfvenic structure, when the spatial spectrum of an incident Alfven wave beam contains a wide set of scales. In this case the spatial spectrum of an incident Alfven structure comprises both small-scale harmonics which effectively excite potential drop variations and large-scale harmonics which penetrate to the ground without substantial attenuation. Specific quantitative estimates require numerical modeling that we plan to perform elsewhere.

The amplitude of the oscillations of the potential drop across the AAR is determined by the parameter $K_{\Phi}=$ $\mu_{0}^{-1} \sqrt{Q / \Sigma_{A}}=\lambda_{A} V_{A}$ (24). The relevant parameters vary over a wide range: $Q \simeq 10^{7}-10^{9} \Omega \cdot \mathrm{m}^{2}, \Sigma_{A} \simeq 0.2-1.0 \Omega^{-1}$, hence $K_{\Phi} \simeq(3-50) \mathrm{V} / \mathrm{nT}$. The additional electron energy gain $e \Delta \Phi$ induced by an Alfven wave with magnetic component $B_{M}^{(0)}=100 \mathrm{nT}$ as follows from (24) for $\lambda_{A}=10 \mathrm{~km}$, $V_{A}=5 \times 10^{3} \mathrm{~km} / \mathrm{s}$, and $T_{\Phi}=1$, can reach up to $5 \mathrm{keV}$.

More complicated spectral features of precipitation and magnetic pulsations are to be observed in the frequency band around the eigenfrequencies of the AAR-associated resonator. The Alfven frequency of the RAAR $f_{A} \simeq V_{A} / 2 d_{R} \simeq$ $0.4 \mathrm{~Hz}$ for the given values of $V_{A}$ and $d_{R}$. Thus, the lowest eigen frequency of the resonator is expected to be $\simeq 0.2 \mathrm{~Hz}$. These estimates have been made assuming a homogeneous altitude profile $V_{A}(z)$ inside layers. In a realistic magnetosphere, the height-distribution $V_{A}(z)$ can be very inhomogeneous. Steep variations of $V_{A}$ may produce a partial reflection of Alfven waves, as stems from (13). This reflection in the upper ionosphere enables the formation of the ionospheric Alfven resonator (IAR) (Lysak, 1988; Belyaev et al., 1990; Pokhotelov et al., 2000). Though our model neglects these features, assuming that the frequencies under consideration are lower than typical IAR frequencies, $\sim 1 \mathrm{~Hz}$, in a realistic auroral region more complicated wave structures may occur combining the IAR and RAAR.

In the high-frequency range riometric and magnetic oscillations are difficult to reveal. Moreover, many approximations used in our analysis (e.g. conditions (2), (9)) reach the limits of their range of validity. Nevertheless, it would be worthwhile to validate the theoretical predictions based on a simplified model. In the frequency range around fractions of a $\mathrm{Hz}$ an oscillatory frequency dependence of the absorption and magnetic spectra is to be observed. The maxima of the coefficient of Alfven wave transmission into the AAR, $T_{\Phi}$, and modulation of the field-aligned potential, $\Delta \Phi$, are to occur at mid-resonant frequencies. At the same time, the maxima of the magnetospheric Alfven wave reflection, $R_{M Q}$, the excitation rate of the RAAR, $T_{R}$, and the ground magnetic signal, $\propto T_{R}$, are to be observed at resonant frequencies of the RAAR. Thus, quasi-periodic variations detected by ground-based magnetometers and riometers are to be shifted by $\sim f_{A} / 2$ in frequency.

Some indications of the predicted behavior can be found analyzing the ground, satellite and rocket observations. 
Thus, Hargreaves et al. (2001) reported the presence of fine structure within an auroral absorption spike event. In addition to significant modulations with periodicities in the $\mathrm{Pi} 2$ band, considerably weaker 5-10 s pulsations appeared in both the absorption and the magnetic records, but with no obvious connection between them. Sato et al. (2002) carried out a direct comparison of pulsating auroras observed from the ground and onboard the FAST satellite $\left(\sim 3 \cdot 10^{3} \mathrm{~km}\right.$ altitude). The aurora appeared as east-west-aligned bands oscillating with a period of $\sim 5 \mathrm{sec}$, observed in the region of the upward field-aligned current. The anti-correlation of ion flux modulation with the down-going high-energy electron flux modulation suggested that the pulsating aurora was caused by a modulation of the field-aligned electric field located above FAST, but far from the equatorial plane. Ivchenko et al. (1999) observed quasi-periodic ( $f \simeq 0.6 \mathrm{~Hz}$ ) structure by magnetic, electric, and particle instruments on sounding rocket (apogee of over $500 \mathrm{~km}$ ) at the border of an auroral arc. The Alfven-type oscillations were accompanied by modulations of field-aligned electron precipitation of the same frequency, indicating acceleration by the wave-related $E_{\|}$. The scale of these waves in the transverse direction $(\simeq 7 \mathrm{~km})$ was much higher than the electron inertial length ( $\simeq 0.16 \mathrm{~km}$ ) for the observed density, which made the possibility of electron acceleration by DAW doubtful. The modulation effects observed in the above experiments fit well the mechanism of the AAR modulation by Alfven waves. The highlighting of the detected wave period could be related to the occurrence of the AAR-associated resonator.

An Alfven wave incident on the AAR from the magnetosphere partially reflects back and partially penetrates into the AAR. Our consideration has shown that the rate of wave reflection/transmission and modulation of the field-aligned potential drop were estimated to be critically dependent on the wave transverse scale. New possibilities provided by multibeam imaging riometers (IRIS) have to be exploited for a study of this new mechanism of ULF modulation of particle acceleration in the auroral region. More study with the use of IRIS riometers is necessary to determine the $2 \mathrm{D}$ structure of precipitation patterns and compare it with the scales predicted by the AAR modulation theory.

\section{Conclusion}

The interaction of magnetospheric Alfven waves with the AAR produces a number of interesting effects that may be observed on the ground and in space. Alfven wave structures with transverse scales about $10 \mathrm{~km}$ can penetrate into the AAR and supply energy to electron acceleration. This modulated acceleration can be an additional mechanism for ULF modulated riometer variations, a feature of which is the lack of time delay between the magnetic and riometer variations. Another feature of this mechanism is the critical dependence of the ratio between the magnetic and riometric signals on the transverse scale of the disturbance. Additionally, in the frequency range around fractions of a $\mathrm{Hz}$, the occurrence of the AAR-associated resonator in the topside ionosphere will induce an oscillatory frequency dependence of modulation. The maximal amplitudes of riometer variations and ground magnetic variations are to be observed at different frequencies, shifted from each other by about the RAAR eigenfre- quency. The predicted effects are to be searched for in simultaneous data from IRIS riometers and magnetometers.

Acknowledgments. We acknowledge helpful discussions with N. Sato, A. Kropotkin, and I. Alexeev, and useful comments of both referees. This research is supported by the grant INTAS 03-515359 (ENF), grant 04-05-64321 from the Russian Fund for Basic Research (VAP), NSF grant OPP-0003881 to the University of Maryland (TJR), NSF grant ATM-0305483 to Augsburg College (MJE), and by a supplement from the NSF Office of International Programs.

\section{Appendix. Analytical Properties of the Reflection and Modulation Coefficients}

The reflection coefficient $R_{M Q}(\bar{\omega})$ as a complex function has interesting features. The function in the right-hand part of (18) is a fractional-linear transform of the variable $u=$ $\exp (2 i \pi \bar{\omega})$. It transforms the axis of real frequencies $\bar{\omega}$ into a circle, symmetrical with respect to the real axis, onto a complex plane of $R_{M Q}$. The absolute value, $\left|R_{M Q}\right|$ should vary between extreme values $\left|R_{M Q}^{(0)}\right|$ and $\left|R_{M Q}^{(1)}\right|$, which are reached at frequencies $\bar{\omega}=n$ and $\bar{\omega}=n+1 / 2$, respectively, whereas $\left|R_{M Q}^{(0)}\right|>\left|R_{M Q}^{(1)}\right|$ for $\tilde{\Sigma}_{P}<1$, and $\left|R_{M Q}^{(0)}\right|<\left|R_{M Q}^{(1)}\right|$ for $\tilde{\Sigma}_{P}>1$. The function $R_{M Q}$ has other properties, namely

$$
\begin{array}{r}
R_{M Q}(\bar{\omega}+n)=R_{M Q}(\bar{\omega}) \\
\operatorname{Im} R_{M Q}(n)=\operatorname{Im} R_{M Q}(n+1 / 2)=0 \\
R_{M Q}(-\bar{\omega})=R_{M Q}^{*}(\bar{\omega}) \\
R_{M Q}(n+1 / 2+\delta \bar{\omega})=R_{M Q}^{*}(n+1 / 2-\delta \bar{\omega}) \\
R_{M Q}\left(\bar{\omega}, \tilde{\Sigma}_{P}\right)=R_{M Q}^{*}\left(\bar{\omega}+1 / 2, \tilde{\Sigma}_{P}^{-1}\right) .
\end{array}
$$

Similar to $R_{M Q}(\bar{\omega})$, the dimensionless coefficient $T_{\Phi}(\bar{\omega})$ is a periodic function of $\bar{\omega}$ with a unit period. The properties (A.1) of the function $R_{M Q}$ are valid for this function as well. We denote $T_{\Phi}(n+1 / 2)=-i \rho_{1}$ and $T_{\Phi}(n)=-i \rho_{2}$, where the real parameters $\rho_{1}$ and $\rho_{2}$ are determined by the relationships

$$
\rho_{1}=\frac{2 \bar{k}_{\perp}}{1+\bar{k}_{\perp}^{2}+\tilde{\Sigma}_{P}} \quad \rho_{2}=\frac{2 \bar{k}_{\perp}}{1+\bar{k}_{\perp}^{2}+\tilde{\Sigma}_{P}^{-1}} .
$$

The function $T_{\Phi}(\bar{\omega})$ transforms a unit interval $0 \leq \bar{\omega}<1$ onto a circle in a complex plane $T_{\Phi}$, with origin in the point $-i\left(\rho_{1}+\rho_{2}\right) / 2$ and radius $\left|\rho_{1}-\rho_{2}\right| / 2$. Therefore the absolute value $\left|T_{\Phi}\right|$ has extreme values $\rho_{1}$ at $\bar{\omega}=n+1 / 2$ or $\rho_{2}$ at $\bar{\omega}=n$.

From (A.2) it follows that for $\tilde{\Sigma}_{P}<1$ the parameter $\rho_{1}>\rho_{2}$, and when $\tilde{\Sigma}_{P}>1$ the reverse inequality is valid, that is $\rho_{2}>\rho_{1}$. When $\tilde{\Sigma}_{P}=1$, these parameters coincide, that is $\rho_{1}=\rho_{2}$, and the coefficient $\left|T_{\Phi}\right|$ does not depend on frequency. The function $\left|T_{\Phi}\left(\bar{k}_{\perp}\right)\right|$ is largest at a critical wave scale $\bar{k}_{*}$ determined by (29). Substituting this value into (A.2) we obtain the maximum possible values of $\rho_{1}=$ $\left(1+\tilde{\Sigma}_{P}\right)^{-1 / 2}$ for $\tilde{\Sigma}_{P}<1$, and $\rho_{2}=\left(1+\tilde{\Sigma}_{P}^{-1}\right)^{-1 / 2}$ for $\tilde{\Sigma}_{P}>1$.

The coefficient $T_{\Phi}$ is related to the $T_{R}$ from $(31,24)$ by the following

$$
T_{R}=1-\frac{i}{2} \bar{k}_{\perp} T_{\Phi}
$$




\section{References}

Arnoldy, R. L., J. L. Posch, M. J. Engebretson, H. Fukunishi, H. J. Singer, and T. Onsager, Pi 1 magnetic pulsations in space and at high latitudes on the ground, J. Geophys. Res., 103, 23581-23591, 1998.

Belyaev, P. P., S. V. Polyakov, V. O. Rapoport, and V. Yu. Trakhtengertz, The ionospheric Alfven resonator, J. Atm. Terr. Phys., 52, 781-788, 1990.

Borovsky, J. E., Auroral arc thickness as predicted by various theories, $J$. Geophys. Res., 98, 6101-6138, 1993.

Borovkova, O. K., O. V. Bol'shakova, L. T. Afanas'eva, N. G. Kleimenova, I. Kangas, and T. Pikkarainen, About different sources of morning and dayside Pi1C pulsations, Geomagn. Aeronomy, 32, 36-41, 1992.

Coroniti, F. V. and C. F. Kennel, Electron precipitation pulsations, J. Geophys. Res., 75, 1279-1289, 1970.

Davidson, G. T., Pitch angle diffusion and the origin of temporal and spatial structures in pulsating aurorae, Space Sci. Rev., 53, 45-82, 1990.

Demekhov, A. G. and V. Y. Trakhtengerts, A mechanism of formation of pulsating aurora, J. Geophys. Res., 99, 5831-5841, 1994.

Engebretson, M. J., L. J. Cahill, Jr., R. L. Arnoldy, S. B. Mende, and T. J. Rosenberg, Correlated irregular magnetic pulsations and optical emissions observed at Siple Station, Antarctica, J. Geophys. Res., 88, 4841-4852, 1983.

Engebretson, M. J., L. J. Cahill, Jr., T. A. Potemra, L. J. Zanetti, R. L. Arnoldy, S. B. Mende, and T. J. Rosenberg, On the relationship between morning sector irregular magnetic pulsations and field aligned currents, J. Geophys. Res., 89, 1602-1612, 1984.

Engebretson, M. J., L. J. Cahill, Jr., J. D. Winningham, T. J. Rosenberg, R. L. Arnoldy, N. C. Maynard, M. Sugiura, and J. H. Doolittle, Relations between morning sector Pi 1 pulsation activity and particle and field characteristics observed by the DE-2 satellite, J. Geophys. Res., 91, 1535-1547, 1986.

Fedorov, E., V. Pilipenko, and M. J. Engebretson, ULF wave damping in the auroral acceleration region, J. Geophys. Res., 106, 6203-6212, 2001.

Grant, I. F. and G. B. Burns, Observations and modeling of correlated $\mathrm{PiB}$ magnetic and auroral luminosity pulsations, J. Geophys. Res., 100, 19387-19404, 1995.

Hargreaves, J. K., A. Ranta, J. D. Annan, and J. C. Hargreaves, Temporal fine structure of nighttime spike events in auroral radio absorption, studied by a wavelet method, J. Geophys. Res., 106 , doi:10.1029/2001JA900008, 2001.

Hughes, W. J. and D. J. Southwood, The screening of micropulsation signals by the atmosphere and ionosphere, J. Geophys. Res., 81, 3234-3240, 1976.

Ivchenko, N., G. Marklund, K. Lynch, D. Pietrovski, R. Torbert, F. Primdahl, and A. Ranta, Quasi-periodic oscillations observed at the edge of an auroral arc by Auroral Turbulence 2, Geophys. Res. Lett., 26, 33653368, 1999.

Janhunen, P., On the current-voltage relationship in fluid theory, Ann. Geophys., 17, 11-26, 1999.

Kinney, R. M., F. V. Coroniti, J. C. McWilliams, and P. L. Pritchett, Mechanisms for discrete auroral arc breakup by non-linear Alfven wave interaction, J. Geophys. Res., 104, 19931-19940, 1999.

Knight, S., Parallel electric fields, Planet. Space Sci., 21, 741-750, 1973.

Lu, G., P. H. Reiff, J. L. Burch, and J. D. Winningham, On the auroral current-voltage relationship, J. Geophys. Res., 96, 3523-3531, 1991.

Lysak, R. L., Theory of auroral zone PiB pulsation spectra, J. Geophys. Res., 93, 5942-5946, 1988.

Lysak, R. L. and C. T. Dum, Dynamics of magnetosphere-ionosphere coupling including turbulent transport, J. Geophys. Res., 88, 365, 1983.

Nakamura, T. S., Parallel electric field of a mirror kinetic Alfven wave, $J$ Geophys. Res., 105, 10729-10737, 2000.

Nose, M., T. Iyemori, M. Sugiura, J. A. Slavin, R. A. Hoffman, J. D. Winningham, and N. Sato, Electron precipitation accompanying Pc5 pulsations observed by the DE satellites and at a ground station, J. Geophys. Res., 103, 17587-17604, 1998.

Olson J. V., Rostoker G., and G. Olchowy, A study of concurrent riometer and magnetometer variations in the Pc4-5 pulsation band, J. Geophys. Res., 85, 1695, 1980.

Olsson, A., A. I. Eriksson, and P. Janhunen, On the current-voltage relationship in auroral breakups and westward-traveling surges, Ann. Geophys., 14, 1265-1273, 1996.

Paquette, J. A., D. L. Matthews, T. J. Rosenberg, L. J. Lanzerotti, and U. S. Inan, Source regions of long-period pulsation events in electron precipitation and magnetic fields at South Pole Station, J. Geophys. Res., 99, 3869-3878, 1994.

Pilipenko, V., M. Vellante, and E. Fedorov, Distortion of the ULF wave spatial structure upon transmission through the ionosphere, J. Geophys. Res., 105, 21225-21236, 2000.

Pilipenko, V. A., E. Fedorov, and M. J. Engebretson, Alfven resonator in the topside ionosphere beneath the auroral acceleration region, J. Geophys. Res., 107, 1257, doi:10.1029/2002JA009282, 2002.

Pokhotelov, O. A., D. Pokhotelov, A. Streltsov, V. Khruschev, and M. Parrot, Dispersive ionospheric Alfven resonator, J. Geophys. Res., 105, 77377746, 2000.

Posch, J. L., M. J. Engebretson, A. T. Weatherwax, D. L. Detrick, W. J. Hughes, and C. G. Maclennan, Characteristics of broadband ULF magnetic pulsations at conjugate cusp latitude stations, J. Geophys. Res., 104, 311-332, 1999.

Rankin, R., J. C. Samson, and V. T. Tikhonchuk, Discrete auroral arcs and nonlinear dispersive field line resonances, Geophys. Res. Lett., 26, 663666, 1999.

Rees, M. H., Auroral ionization and excitation by incident energetic electrons, Planet. Space Sci., 11, 1209-1218, 1963.

Reiff, P. H., G. Lu, J. L. Burch, J. D. Winningham, L. A. Frank, J. D. Craven, W. K. Peterson, and R. A. Heelis, On the high- and low-altitude limits of the auroral electric field region, Auroral Plasma Dynamics, 80, 143-154, 1993.

Sato, N., D. M. Wright, Y. Ebihara, M. Sato, Y. Murata, H. Doi, T. Saemundsson, S. E. Milan, M. Lester, and C. W. Carlson, Direct comparison of pulsating aurora observed simultaneously by the FAST satellite and from the ground at Syowa, Geophys. Res. Lett., 29, 2041, doi:10.1029/2002GL015615, 2002.

Southwood, D. J. and M. G. Kivelson, Charged particle behavior in lowfrequency geomagnetic pulsations. 1. Transverse waves, J. Geophys. Res., 86, 5643-5655, 1981.

Stasiewicz, K., P. Bellan, C. Chaston, C. Kletzing, R. Lysak, J. Maggs, O. Pokhotelov, C. Seyler, Shukla, L. Stenflo, A. Streltsov, and J.-E. Wahlund, Small scale Alfvenic structure in the aurora, Space Sci. Reviews, 92, 423-533, 2000.

Vogt, J., Alfven wave coupling in the auroral current circuit, Surveys of Geophysics, 23, 335-377, 2002.

Vogt, J. and G. Haerendel, Reflection and transmission of Alfven waves at the auroral acceleration region, Geophys. Res. Lett., 25, 277-280, 1998.

Vogt, J., G. Haerendal, and K. H. Glassmeier, A model for the reflection of Alfven waves at the source region of the Birkeland current system: The tau generator, J. Geophys. Res., 104, 269-278, 1999.

Weimer, D. R., D. A. Gurnett, C. K. Goertz, J. D. Menietti, J. L. Burch, and M. Sugiura, The current-voltage relationship in auroral current sheets, $J$. Geophys. Res., 92, 187-194, 1987.

Wright, A. N., W. Allan, M. S. Ruderman, and R. C. Elphic, The dynamics of current carriers in standing Alfven waves: Parallel electric fields in the auroral acceleration region, J. Geophys. Res., 107, 10.1029/2001JA000231, 2002.

Yoshikawa, A. and M. Itonaga, Reflection of shear Alfven waves at the ionosphere and the divergent Hall current, Geophys. Res. Letters, 23, 101-104, 1996.

E. Fedorov (e-mail: fedorov@ifz.ru), V. Pilipenko (e-mail: pilipenk@ augsburg.edu), M. J. Engebretson (e-mail: engebret@augsburg.edu), and T. J. Rosenberg (e-mail: rosenberg@uarc.umd.edu) 